\title{
Horizontal daylighting system for office buildings
}

\author{
Daniel Vázquez-Molinía ${ }^{a}$, Mario González-Montes ${ }^{\mathrm{a}, *}$, \\ Antonio Álvarez Fernández-Balbuena ${ }^{a}$, Ángel García-Botella ${ }^{\mathrm{b}}$, \\ Wilfried Pohl ${ }^{\mathrm{c}}$, Teresa Galan ${ }^{\mathrm{d}}$, Eusebio Bernabéu ${ }^{\mathrm{a}}$ \\ a Universidad Complutense de Madrid. Dpto. Óptica, Avda Complutense S/N, 28040 Madrid, Spain \\ b Universidad Politécnica de Madrid. Dpto. Física aplicada a los recursos naturales, Ciudad, Universitaria S/N, 28040 Madrid, Spain \\ ${ }^{\mathrm{c}}$ Bartenbach LichtLabor GmbH. Rinner Straße 14, 6071 Aldrans, Austria \\ d Lledó Iluminación, Cid Campeador 14, 28935 Mostoles, Spain
}

\section{A R T I C L E I N F O}

\section{Article history:}

Received 25 February 2013

Received in revised form 6 June 2013

Accepted 21 August 2013

\begin{abstract}
A B S T R A C T
This work is focused on a sunlight collection device for solar lighting systems aimed at non-residential buildings. The design takes advantage of solar energy that reaches the facade of the building for lighting purposes, thereby reducing building energy consumption by supplementing it with clean, renewable energy. This daylighting system's structure comprises a light collector located outside the vertical facade of the building, a modular light guide that runs along the horizontal false ceiling and light extractor luminaires strategically positioned along the guide. The system is configured specifically to the particular characteristics of each building (façade orientation, latitude and longitude) to optimize the global performance. The present paper describes in detail the anidolic collecting system as a part of the comprehensive daylighting system. However the entire daylighting system has been completely developed and experimentally tested, and its prototype is currently integrated into Lledó S.A company's facilities.
\end{abstract}

(C) 2013 Elsevier B.V. All rights reserved.
The collecting system, based on anidolic optical components, is responsible to collect and redirect sunlight. An innovative collector design minimizes the dependence of its behavior regarding solar incidence angle. In order to obtain the minimum level of illumination in an office area, an illuminance of at least $300 \mathrm{~lx}$ on the work plane should be assured. The whole system provides a lighting level of 300-400 lx during the mid-time work hours in an area of $4 \mathrm{~m} \times 10 \mathrm{~m}$, i.e. $40 \mathrm{~m}^{2}$. The sun-luminaires manage to reduce glare (interference with vision or eyestrain) while maintaining an optimal distribution.

\section{Introduction}

Nowadays it's no denying the growing awareness of renewable energy systems applied to the domain of buildings. The change in mentality and economic strategies for all agents involved in this industry becomes a necessity that should add the appropriate training for material handling, construction and energy systems compatible with conservation and environmental degradation. Thus the United Nations Program for Environment in the “Global Green New Deal” March 2009, the European commission

\footnotetext{
* Corresponding author. Tel.: +34 679420991; fax: +34 913946885

E-mail address: mariog@fis.ucm.es (M. González-Montes).
}

"Greenlight" project March 2006 and the "Greenbook" founded by the commission of European communities March 2006, are some a clear examples of this trend.

The benefits of daylight in office buildings are well known and actually nobody questions that point [1-3]. The use of daylighting reduces the maintenance cost and improves the well-being of building occupants. The productivity of workers rises and the work environment is healthier. Improving the welfare of building users affects themselves and thus to the company and its owners who see improved performance of their workers [4,5]. In fact the effects of daylight systems on building users should be an important concern for building design due it means satisfaction for both; building occupants, owners and by extension the whole society. As a requirement to provide visual comfort conditions in rooms it becomes mandatory to ensure certain values and limits for the illumination level, luminance, glare index and color composition. The required level may vary as a function of the activities carried out in each room [6].

At the present time everybody is involved to reduce the $\mathrm{CO}_{2}$ emissions and therefore it's mandatory to save electrical energy. In Spain for example the consumption of lighting task in commercial buildings reaches a $22 \%$ of the total energy consumption, and the law fixes a minimal efficiency for this kind of buildings (Technical code for buildings, Spanish BOE 28 march 2006. Real decree 1369/2009 ecologic design for energy devices, Directive CE91-2002 building energetic efficiency). 
Non-imaging optics systems are suitable solutions when high concentration factors and high efficiency are desirable, anidolic (without-image) based systems have proved a great potential in the field of daylighting systems although commercial solutions that are not yet widely available mainly due to its elevated manufacture and installation costs. Anidolic daylighting systems applied to buildings can be extensively found in literature (LESO, Solar Energy and Building Physics Laboratory) together with its own design criteria, building's façade-integrated applications, simulation tools and results [7].

This work aims to harness the light reaching the building façade for lighting purposes. The system is devised to adapt itself to the characteristics of a specific building, although its performance could be improved if the building planning would take into account the lighting system integration and restrictions. Some studies suggest that the way to optimize the building's use of solar energy should be planned in initial stages of construction [8].

Present work is comprehended in ADASY (Active Daylighting System) project and although the whole daylighting system is already developed and its prototype is currently integrated into Lledó S.A company's facilities, this study will focus mainly on the collecting system, detailing its design and optimization process. The problem will be addressed regarding the specific functions assigned to the constituent parts thereof. The analysis of the lighting system will be divided into: collection, transmission and distribution of light.

\subsection{Collecting system}

The collector has two main functions, the first to collect the maximum possible light flux and the second, and not least, to direct the light with a given orientation into the duct of high reflectivity. It is a priority to reduce the angle for output light of the caption system which will be based on a T-CPC (Truncated Compound Parabolic Concentrator) matrix geometry.

Further analysis has been developed to test the collecting system's performance for the local conditions of solar irradiance in the city of Madrid. The innovation of this collecting system resides in optimizing both the geometry of the unit cell CPC as well as the forming matrix parameters as: compact ratio and tilt angle.

\subsection{Transmission}

Once the incident sunlight is captured, the flux is redirected to room zones distant from the window where the sunlight can't reach directly. This is accomplished by horizontal light guides inside building's false ceiling. The light is channeled inside these high reflectivity mirrored devices through reflection on their inner faces.
A study of propagation of light in this medium will be shown, quantifying the losses that will be essential to design the subsequent extraction and distribution system.

\subsection{Distribution}

Finally a system for light extraction/distribution will be devised that aims to spread the sunlight efficiently and balanced into the room. This task will approve various extraction devices to work for a wide range of irradiance conditions and will be complemented with materials of different roughness to avoid "hot spots" or excessive levels of light. These sun luminaires will also be designed to minimize glare.

\section{Sunlight collecting system}

The first component is the collecting system, based on anidolic optical components, which is responsible to collect and redirect sunlight. This is a key component since it must operate with high numerical aperture, high efficiency and finally the output beam of the collecting system must have divergence lower than $30^{\circ}$ for further guidance, reducing this way reflection losses and achieve maximum efficiency.

The collector structure is based on the parabolic component called T-CPC (Compound Parabolic Concentrator Truncated). Active components are discarded in our collecting because the product price and maintenance would increase; in addition the complexity of the system would rise. CPC are non-imaging concentrators (anidolic devices). These have the capability of reflecting all of the incident radiation within wide limits, increasing the exploitable incident radiation. Their potential as collectors of solar energy is well known and was noted by Winston [9], Joseph J. O'Gallagher [10] or Sansoni [11] among others.

With the purpose of extending the capture solar angle, an operation known as truncation is carried out in the collector. This involves cutting the input (input area) of the CPC by a cutting plane determined by an inclination angle alpha, increasing the energy gathered monthly and yearly. Furthermore there is another parameter, the tilt angle $\beta$ between the horizontal axis and the axis of symmetry of the CPC, which together with alpha, $\alpha$, allows facing the entrance of $\mathrm{CPC}$ to the plane of maximum solar irradiance. This operation defines a new geometry known as T-CPC (CPC Truncated). A more thorough study of this and other parabolic concentrators may be reviewed in [12] (Fig. 1).

Another advantage of the T-CPC consists in the implementation of the truncation angle $\alpha$, thus the T-CPC elements may form an array whose input surface would have the desired orientation and the T-CPC forming the collector will not cast a shadow over the rest

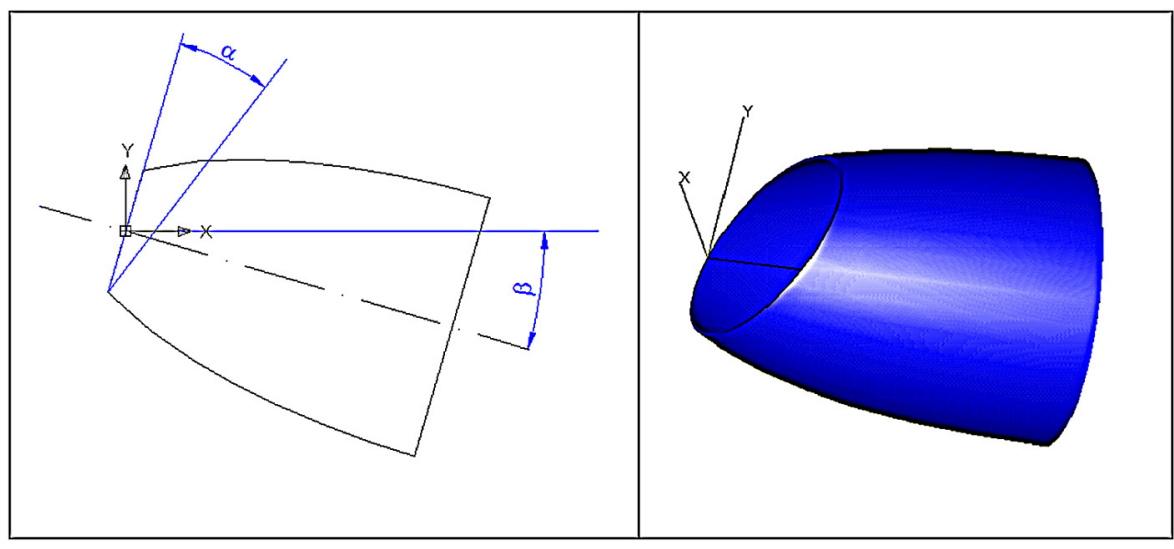

Fig. 1. T-CPC. $\alpha$, Truncation angle. $\beta$, slope angle. 

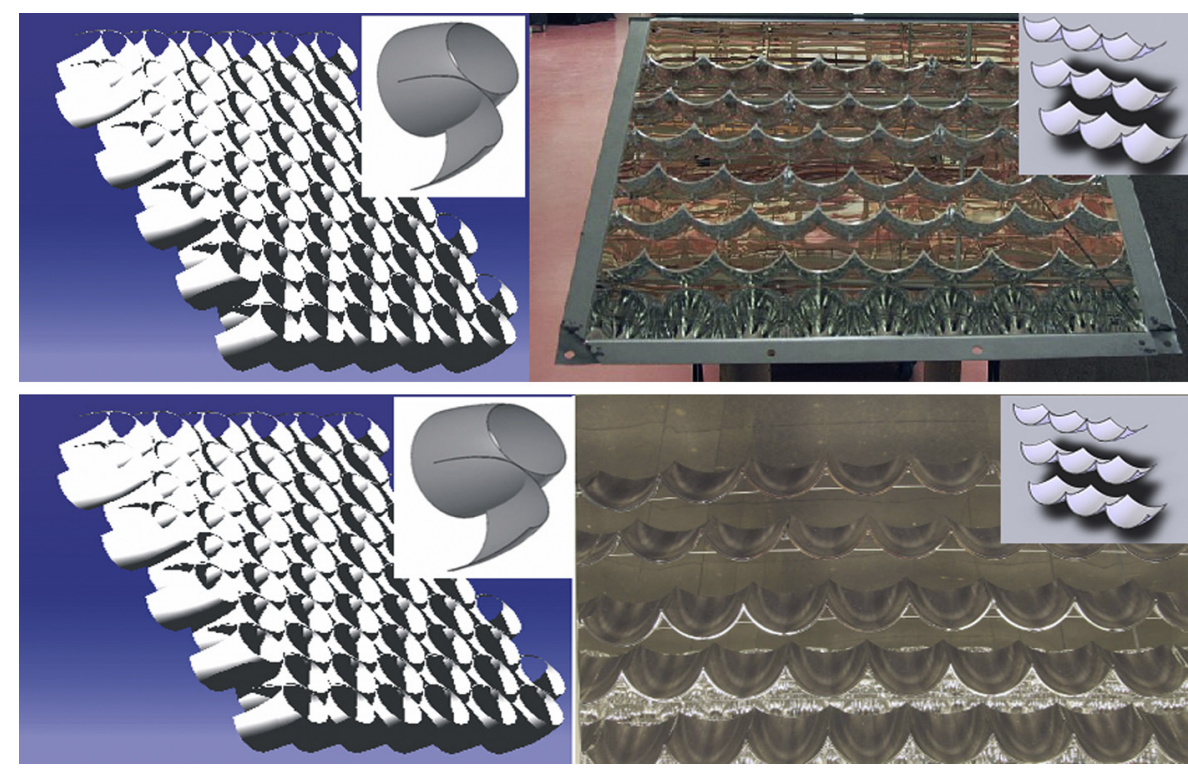

Fig. 2. Truncated $\mathrm{CPC}(\mathrm{T}-\mathrm{CPC})$ (left) and prototype (right) array collectors.

components of the collecting matrix, without harming the acceptance angle of the entire system. The orientation of a CPC collector is related to its acceptance angle [13].

The optical component of the collector system consists of a 3D matrix processed by PVD metallization. The PVD technique (Physical Vapor Deposition) involves the formation of a vapor to be deposited. It starts directly from the solid material to be deposited to turn it into a vapor by heating or ion bombardment energy. The vapor formed condenses on the substrate surface forming a thin layer of high specular reflection.

Considering this matrix structure we have to introduce a new parameter, the compaction rate. The aim is to reduce the gap (useless area) between T-CPC's without drastically altering the operation of the collecting system; aperture and output angle, efficiency, flux distribution etc (Fig. 2).

In order to optimize the performance of the collector a depth structural and functional analysis has been performed by the Austrian company Bartenbach L'chtLabor. Several models based on the initial T-CPC geometry are obtained. One of these optimized T-CPCs, shown in Fig. 2, forms the prototype's collecting system and will be named as VarG_12 model. This T-CPC model is obtained by the optimization of the T-CPC toward a lama type structural unit that reduces reflections to increase the collecting efficiency. This type of collector will be compared to the T-CPC original matrix along with several different optimized models. These modifications on the structural unit may improve the performance and efficiency of the collecting matrix at the expense of losing (in some cases) output flux uniformity in the working range (Fig. 3).
The optimization parameters used are the tilt angle of the inlet glazing $\boldsymbol{\alpha}$ (CPC truncation angle), the tilt angle of the T-CPC $\boldsymbol{\beta}$, the radiation angle $\boldsymbol{\theta}$ and the compaction ratio $(\mathbf{d x}, \mathbf{d y})$, apart from a shift distance of the CPC parabola from the axis. These degrees of freedom provide optimization parameters and the system evolves to a collector model which can capture a greater amount of daily radiant energy.

The calculation of the target function consists of several steps:

- angular dependent transmission of the T-CPC matrix + horizontal light guide is determined by ray tracing (commercial software, in this case ASAP from Breault Research)

- the flux at the end of the light guide coming from direct sunlight is calculated with a program developed by Bartenbach LichtLabor for fixed time steps in a whole year and thus the available quantity of light [ $\mathrm{klm} \mathrm{h}$ ] during work hours is determined

- different target functions have been implemented based on these data (efficiency, uniformity of flux in the course of the days) and optimal solutions concerning these target functions have been found by local minimizations using different, adeptly chosen starting points

The calculation for a whole year at fixed time steps is done according to DIN 5034-2 and depends on

- geographical location of the site (latitude, longitude, altitude)

- orientation of the facade
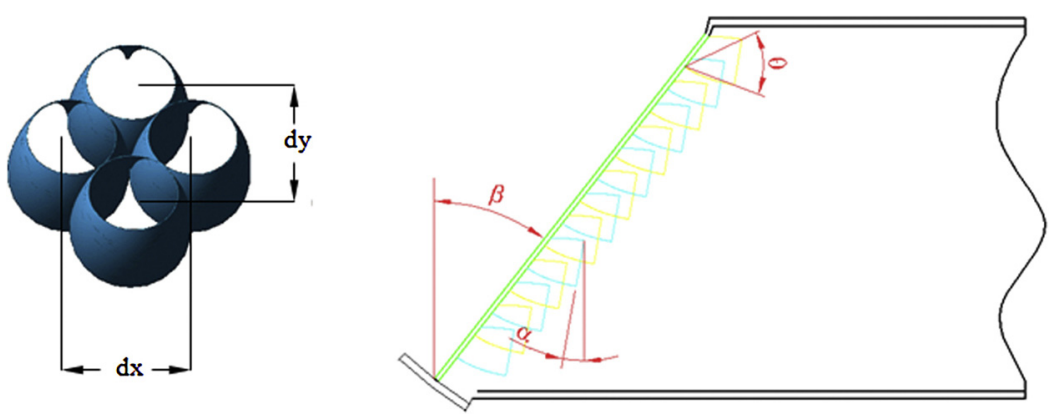

Fig. 3. Optimization parameters. 
- monthly averaged sun shine probabilities

- monthly averaged Linke turbidity factors $T_{L}$ available e.g. from SoDa http://www.soda-is.com/

Alternatively half-hourly climate data from S@tel-Light http://www.satel-light.com/available for the years 1996-2000 have been used.

Both calculations methods have been applied to four towns in Spain for a south facade:

- Madrid

- Sevilla

- La Coruña

- Barcelona

Different orientations of the facade have also been tested but the south facade was of primary interest. For many hundreds of adeptly chosen starting points the results of local minimizations using e.g. downhill-simplex algorithm (Nelder-Mead) have been compared. The aim is to have a high light quantity during work hours and at the same time to reduce the differences in available flux in the course of a year. This is a compromise between two different aspects that favor other types of T-CPCs.

The target functions have shown similar behavior for both methods and for all four towns. Thus for the final optimizations the focus has been on Madrid and the calculation method using S@tel-Light data. After finding an optimal geometry for the collecting system including the transport into the depth of the building using a horizontal light guide, sun-luminaires have to be developed that spread the sunlight into the rooms. These luminaires have to provide good lighting quality (e.g. avoiding glare by sun luminance) and are not part of the optimization process for the T-CPCs.

In comparison to other anidolic collectors (e.g. LESO's Anidolic Solar Blind) these T-CPCs are especially designed to transfer direct sun light deep into a building using a horizontal light guide (Fig. 4).

This optimization process aims to achieve the greatest amount of input radiant energy. However it is advisable to balance the energy flux and the CPC emission angle so the distribution losses could be minimized and the extraction process would tend to be more uniform and efficient as the radiation angle decreases. This will take another series of optimizations to address not only energy efficiency in the distribution of light energy eta*, but also

1-VARN_02
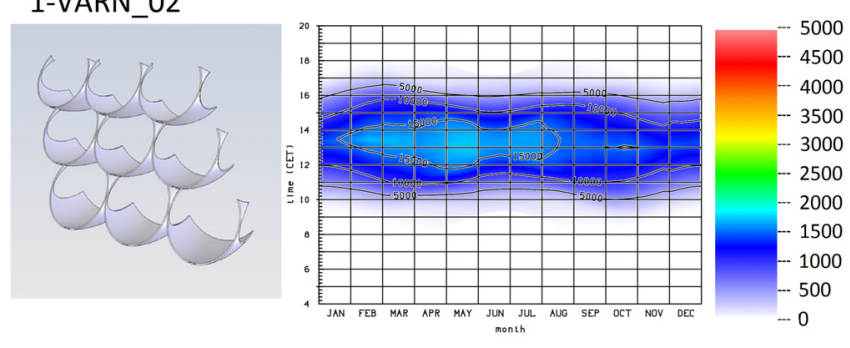

2-VARG_12
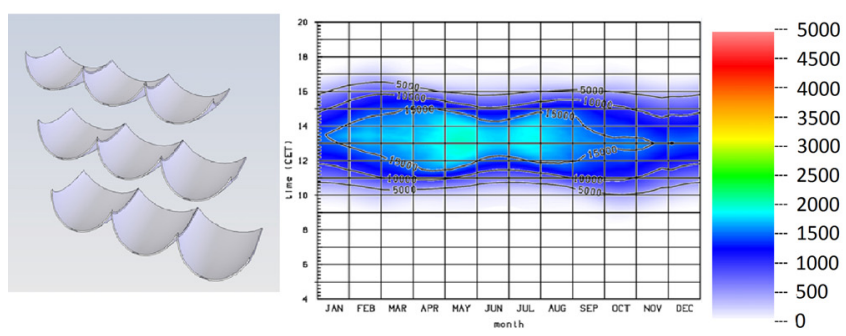

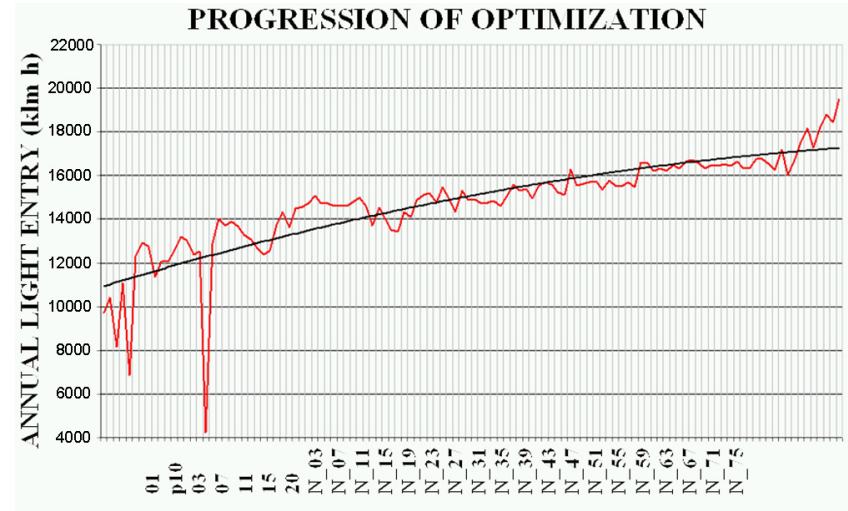

COLLECTING MODEL VARLATION

Fig. 4. Optimization progress.

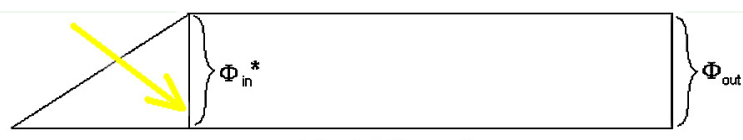

eta ${ }^{*}: \frac{\Phi_{\text {out }}}{\Phi_{\text {in }}^{*}} \quad$ uniformity $u: u=\frac{\text { minimal daily maximum }}{\text { maximal daily maximum }} \quad$ rating $r: r=\frac{\text { eta }^{*}+u}{2}$

Fig. 5. Optimization target.

considering the uniformity of incident light along the day $\mathbf{u}$, and assessing both variables together to define the target (rating) of optimization $\mathbf{r}$ (Fig. 5).

Starting from this point the individual analysis of the CPC will be reviewed leading to a globally optimized collection system that ensures both; an efficient reflective geometry and an ideal location and orientation for each structural unit based on the T-CPC.

As seen in Fig. 6 the geometry of the structural unit of the collector array evolves from the classic CPC design to parabolic slat geometry. This modification is due to the integration of the T-CPC within a matrix where the surrounding parabolic concentrators are also capable of redirecting the flux properly intended to be reflected at the initially isolated $\mathrm{CPC}$, therefore, and considering the solar incident positions related to working hours, the total surface of the collector can be reduced improving efficiency (less reflection losses) without seriously harming uniformity.

\section{3-VARN_29}
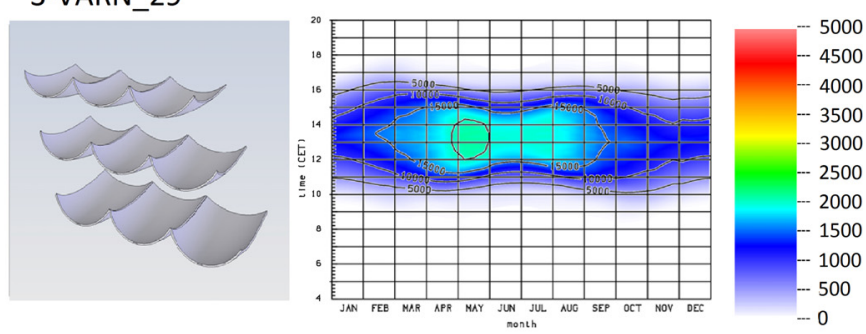

4-VARB_29

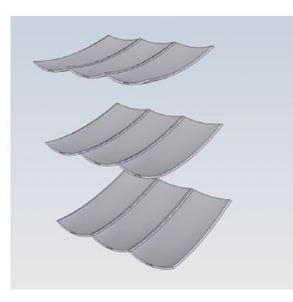

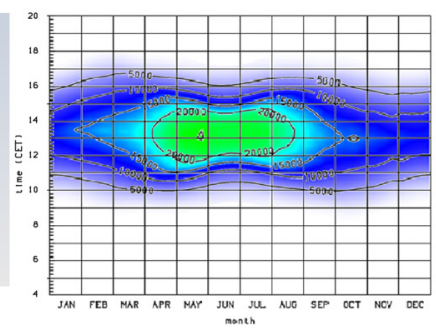

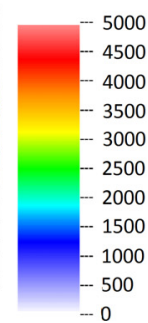

Fig. 6. Collector optimized models and collected luminous flux diagrams. 


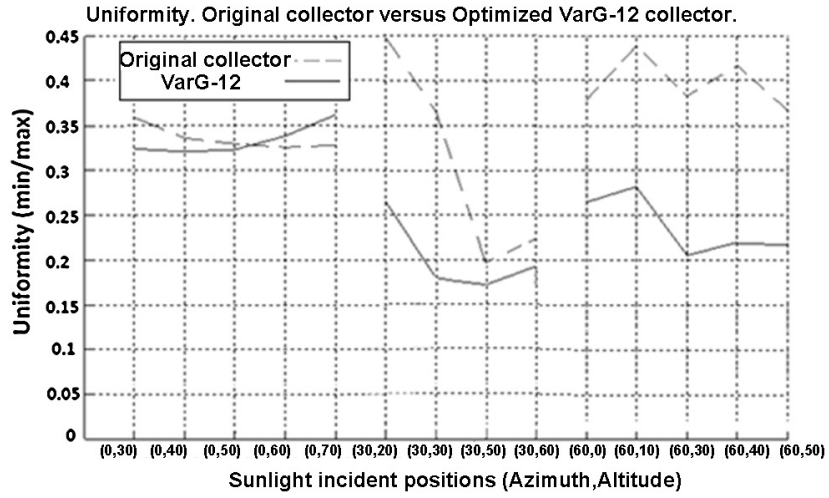

Fig. 7. Uniformity response: original collector vs. VarG-12 collector.

At this point it is necessary to compare performance between the original collector versus optimized models, and thus analyze the variation in performance resulting by the optimization process. Finally the balance between the new conditions of uniformity and efficiency must be evaluated, in order to decide the validity of the new collector obtained.

From here on, the analysis will consider the system as a whole (not just the collector and the light guide) and thus will take into account the behavior of extraction systems (luminaires) and its balance with the collector model chosen as well as the other system's configuration parameters. Furthermore, the simulations carried out, which provide information regarding uniformity and illumination level at the work plane, will be compared with experimental results provided by the real prototype.

Below are the results obtained by comparing the uniformity at the work plane (minimum/maximum luminance value) for two collector models, the original collector and the VarG-12 model (Fig. 7):

As expected the original collector shows a more uniform behavior as the greater amount of reflective entry surfaces leads to an increase of the number of incident light reflections and consequently produces a greater randomization of the light's propagation angle whose flux is distributed more evenly through the work plane, nevertheless its efficiency is lower than in the improved model.

Anyway VarG-12 model, despite losing uniformity level, provides acceptable values that can be enhanced by rough surfaces in both the light guide and extraction systems. The pair distributionefficiency will be always evaluated considering the overall system (collector, guide and luminaries) to assess the benefits of a particular collection system and the desirability of supplementing it with additional complements (diffusers, channels, micro-roughness, diffractive networks) that may modulate the daylight system's global behavior. Thus the level of uniformity of the collecting system presented in the figure above is potentially improved, since it is obtained for a basic configuration of the lighting system and can be modified by adding spot-breaking elements cited previously.

A first prototype of this T-CPC VarG-12 has been manufactured by injection molding and evaporating. The size of the light guide has been $5.79 \mathrm{~m} \times 0.57 \mathrm{~m} \times 0.3 \mathrm{~m}$ (length $\times$ width $\times$ height). Using these prototype measurements for typical sun positions have been performed to verify the theoretical simulations based on forward ray tracing. At the same time the practicability of this daylight system has been evaluated by integrating first design examples of sun-luminaires.

The CIE (International Commission on Illumination) promotes through a dedicated set of recommendations [14] uniformity of illumination on the work plane as an important parameter to ensure the well-lit space and avoid visible light deficit. Moreover,

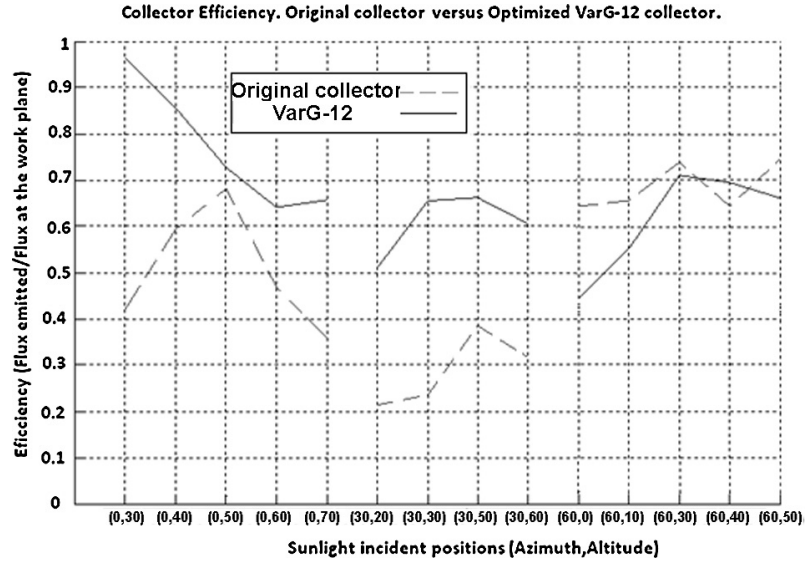

Fig. 8. Efficiency level: original collector vs. VarG-12 collector.

considering health and psychological benefits of daylighting systems, complementary subjective criteria must be also applied (Collins, 1975, 1976).

To establish a quantitative criterion for uniform lighting thresholds a series of indications arising from technical guides and codes regarding lighting task in workplaces $[15,16]$ are significantly consistent, which recommend that the ratio between the minimum and maximum lighting levels in the area of the position where the task is done is not less than 0.8 .

The uniformity criterion should not be so rigorous when applied to daylighting systems as in the case of artificial lighting, the variable nature of solar light suggest an inherent and unpredictable advance to uniformity. The balanced distribution of daylight may lead to a reduction of the minimum illuminance acceptance threshold as several studies support this theory [14,17]. The spectral quality of light may compensate for the lower illuminance obtained at certain zones of the light distribution that would drop the uniformity ratio.

Ultimately the levels of ADASY uniformity are positive for a natural lighting system although the values of this parameter are about a half of the requirements for the lighting systems in offices but keeping in mind that ADASY is designed to complement a conventional electric lighting system.

In addition the data obtained about its efficiency are very promising and ensure optimum use of natural light falling on the building's facade. The following figure shows again a comparison between the two models studied collector but this time in terms of efficiency (Fig. 8):

Following the outcome of the last figure collector checks the advantages of Var-G12 compared to the original model. Optimized VarG-12 model collector shows superior efficiency for almost all of the analyzed solar incidence angles. This improvement in efficiency validates the optimization process and, after comparing theVarG12 model with the rest of the optimized collector, we obtain enough arguments to choose the model Varg-12 as the definitive collector to integrate into ADASY system.

Once the collector model is chosen and so the configuration parameters are set it's possible to measure the system's performance in terms of illumination level. Through the simulations carried out with ray tracing program "TracePro" and comparing these estimations with experimental results generated by the system's prototype, measured in Bartenbach facilities, it can be assured a lighting level of 300-400 lx during the mid-time work hours in an area of $4 \mathrm{~m} \times 10 \mathrm{~m}$, i.e. $40 \mathrm{~m}^{2}$.

In order to go into detail about the integration of the collecting system into the prototype, next figure shows an outline of the glazing and the relative position of the collector array (Fig. 9). 


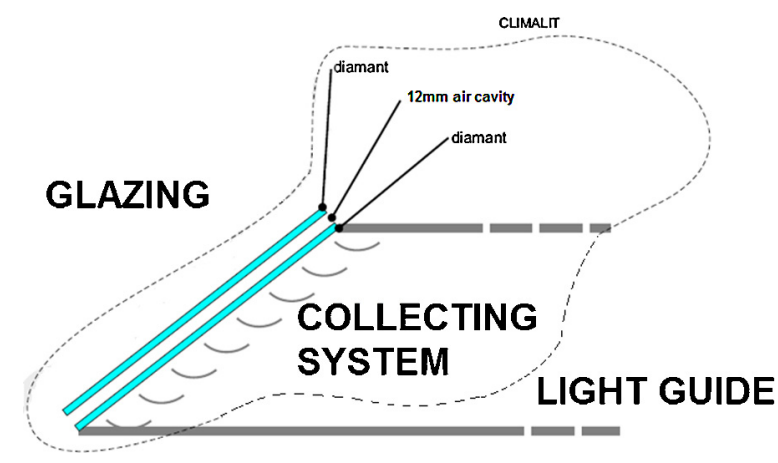

Fig. 9. Glazing scheme. Collecting system integration.

The prototype substrate is plastic and mirror coating. System dimensions are $0.5 \mathrm{~m} \times 1.1 \mathrm{~m} \times 11 \mathrm{~m}$, although the prototype has scaled to half its size. The closing glass that separates the outer collector system has double glazing to prevent condensation and maintain good isolation. Within the light guide, and after closing, is situated the TCPC matrix. The light guide is made of high reflectance and is coupled to the external closing. Since the conjunct closing-collector-guide is totally sealed the maintenance is extremely simply and only requires periodic cleaning of the glass tilted closing.

\section{Conclusions}

An innovative horizontal daylighting system of demonstrated efficiency and balanced flux distribution has been designed. ADASY is able to illuminate rooms of $40 \mathrm{~m}^{2}$ per system . The modular topology of this day lighting system provides it with great flexibility that makes it suitable for any office structure or non-residential buildings, as well as its installation is customized for each specific building where it intended to be integrated, analysing environmental, geographical and construction factors, therefore ensuring optimal behavior.

As a result of the optimization process different collector models designs based on the original CPC are reached, these collecting arrays increase the energy transferred to the lighting system and also improve the light's flux uniformity. This way more than $15,000 \mathrm{klm}$ h/year solar collection can be, and the final ADASY configuration (including diffusers materials and micro-roughness surfaces) is able to maintain a daily uniformity ( $\mathrm{min} / \mathrm{max}$ ) factor above 0.5.The behavior of the collector ADASY ensures enough flux transferred to the rest of the system and maintains a balanced flux delivered during working hours.

The day lighting system is based on a T-CPC collection system that minimizes the system's dependence on solar incidence which means an appropriate behavior for virtually any time of year during working hours. Moreover the controlled aperture angle, limited by the collector array, reduces reflection losses.

\section{Acknowledgements}

ADASY is an European EUREKA 3575 Project leading by Lledó Group which has been partially supported by CDTI and IMADE. UCM, UPM and Bartenbach Licht Labor have contributed to the optical design of the daylighting system.

\section{References}

[1] C. Luo, To Capture the Sun and Sky "Lighting Futures", vol. 1, 4 ed., Polytechnic Institute Lighting Research Center, Rensselaer, NY, 1998.

[2] J.H. Heerwagen, J.A. Johnson, P. Brothers, R. Little, A. Rosenfeld, Energy effectiveness and the ecology of work: links to productivity and well-being, in: Proceedings of the 1998 ACEEE Summer Study, Washington, DC: The American Council for an Energy-Efficient Economy, 1998, pp. 8.123-8.132.

[3] G. Franta, K. Anstead, Daylighting Offers Great Opportunities, Window \& Door Specifier-Design Lab, Spring, 1994, pp. 40-43.

[4] J. Pierson, If sun shines in, workers work better, buyers buy more, The Wall Street Journal (1995) B1-B7 (20.11.1995).

[5] Pape, W.R. "At What Cost Health? Low Cost, As It Turns Out.” Inc. Online. http://www.inc.com/extra/stories/06169821.html. accessed 12.08.1998).

[6] G.K. Oral, A.K. Yener, N.T. Bayazit, Building envelope design with the objective to ensure thermal, visual and acoustic comfort conditions, Building and Environment 39 (2004) 281-287

[7] F. Linhart, K.F. Wittkopf, J.-L. Scartezzini, Performance of anidolic daylighting systems in tropical climates-parametric studies for identification of main influencing factors, Solar Energy 84 (2010) 1085-1094.

[8] J.H. Kampf, D. Robinson, Optimization of building form for solar energy utilization using constrained evolutionary algorithms, Solar Energy and Building Physics Laboratory, Station 18, Ecole Polytechnique Fédérale de Lausanne, 1015 Lausanne, Switzerland, 2010.

[9] R. Winston, Solar concentrators of novel design, Solar Energy 16 (1974) 89-95

[10] J.J. O'Gallagher, Nonimaging Optics in Solar Energy, 2008, ISBN 9781598293302 .

[11] P. Sansoni, D. Fontani, F. Francini, D. Jafrancesco, G. Longobardi, Optical design and development of fibre coupled, compact solar collectors, Lighting Research and Technology 39 (2007) 17-30, 1.

[12] A. García-Botella, A.A. Fernández-Balbuena, E. Bernabéu, D. Vazquez, A. Gonzalez-Cano, Hyperparabolic concentrators, Applied Optics 48 (4) (2009).

[13] A. Soteris, Kalogirou, Solar thermal collectors and applications, Progress in Energy and Combustion Science 30 (2004) 231-295.

[14] CIE, Anon. CIE Publication, No. 29.2, Guide on interior lighting (1986).

[15] The technical guide for assessing and preventing risks related to the use of work places (2006).

[16] CIBSE, Code for interior lighting (1994).

[17] M. Fontoynont, Perceived performance of daylighting systems: lighting efficiency and agreeableness, Solar Energy 73 (2) (2002) 83-94. 